\title{
Optical Quantal Analysis Indicates That Long-Term Potentiation at Single Hippocampal Mossy Fiber Synapses Is Expressed through Increased Release Probability, Recruitment of New Release Sites, and Activation of Silent Synapses
}

\author{
Christopher A. Reid, ${ }^{1 \star}$ Don B. Dixon, ${ }^{1 *}$ Michiko Takahashi, ${ }^{1}$ Tim V. P. Bliss, ${ }^{1}$ and Alan Fine ${ }^{1,2}$ \\ ${ }^{1}$ Division of Neurophysiology, National Institute for Medical Research, London NW7 1AA, United Kingdom, and ${ }^{2}$ Department of Physiology and \\ Biophysics, Faculty of Medicine, Dalhousie University, Halifax, Nova Scotia B3H 4H7, Canada
}

It is generally believed that long-term potentiation (LTP) at hippocampal mossy fiber synapses between dentate granule and CA3 pyramidal cells is expressed through presynaptic mechanisms leading to an increase in quantal content. The source of this increase has remained undefined but could include enhanced probability of transmitter release at existing functional release sites or increases in the number of active release sites. We performed optical quantal analyses of transmission at individual mossy fiber synapses in cultured hippocampal slices, using confocal microscopy and intracellular fluorescent $\mathrm{Ca}^{2+}$ indicators. Our results indicate that LTP is expressed at functional synapses by both increased probability of transmitter release and recruitment of new release sites, including the activation of previously silent synapses here visualized for the first time.

Key words: quantal analysis; synapse; plasticity; long-term potentiation; silent synapse; hippocampus; calcium; confocal microscopy; dendritic spine; mossy fiber; CA3 pyramidal cell; fluorescence imaging

\section{Introduction}

Hippocampal CA3 pyramidal neurons have two classes of excitatory synaptic inputs that exhibit distinct forms of long-term potentiation (LTP) (Zalutsky and Nicoll, 1990). LTP of associational-commissural and recurrent collateral inputs requires postsynaptic NMDA receptor activation (Bliss and Collingridge, 1993); in contrast, LTP of mossy fiber (MF) input is independent of NMDA receptor activation (Harris and Cotman, 1986; Zalutsky and Nicoll, 1990; Johnston, 1992). Although there is controversy regarding the locus of induction of mossy fiber LTP (Williams and Johnston, 1989; Zalutsky and Nicoll, 1990; Yeckel et al., 1999; Mellor and Nicoll, 2001), there is general agreement that the potentiation is expressed entirely via presynaptic mechanisms. In particular, reduced failure rate, increased

\footnotetext{
Received July 30, 2003; revised Feb. 16, 2004; accepted Feb. 20, 2004

This work was supported by grants from the Human Frontier Science Program, the European Commission Framework V Program, and the Canadian Institutes of Health Research. C.A.R. held a Royal Society Howard Florey fellowship. We thank Gian-Luca Raimondi, Grant Roalfe, Sukhvinder Dhanjal, and the National Institute for Medical Research Mechanical Engineering section for technical assistance.

${ }^{*}$ C.A.R. and D.B.D. contributed equally to this work.

Correspondence should be addressed to Alan Fine, Division of Neurophysiology, National Institute for Medical Research, The Ridgeway, Mill Hill, London NW7 1AA, UK. E-mail: a.fine@dal.ca.

C. A. Reid's present address: Department of Physiology, University of Melbourne, Parkville, Victoria 3010, Australia.

M. Takahashi's present address: Department of Physiology, University College London, London WC1E 6BT, UK. DOI:10.1523/JNEUROSCI.3567-03.2004

Copyright $\odot 2004$ Society for Neuroscience $\quad$ 0270-6474/04/243618-09\$15.00/0
}

coefficient of variance (Xiang et al., 1994; Lopez-Garcia et al., 1996), and reduction in paired-pulse facilitation (Staubli et al., 1990; Zalutsky and Nicoll, 1990; Xiang et al., 1994; Weisskopf and Nicoll, 1995) after MF LTP all indicate an increase in mean quantal content $(m$, the average number of quanta of transmitter released per stimulus). In keeping with an increase in quantal content, the amount of transmitter released has been found by patch sensors to increase with MF LTP (Maeda et al., 1997). The quantal content of transmission at a synapse is determined by the number of releasable quanta, $N$ (which in the CNS may correspond to the number of functional transmitter release sites), and the probability, $p_{r}$, of each quantum being released. In previous electrophysiological studies recording from unknown numbers of synapses, it has been difficult to determine unambiguously which of these quantal parameters is increased in LTP.

To overcome such interpretational difficulties, optical methods can be used to detect synaptically evoked excitatory postsynaptic $\mathrm{Ca}^{2+}$ transients (EPSCaTs; pronounced "ep-scats") in dendritic spines (Muller and Connor, 1991; Malinow et al., 1994; Emptage et al., 1999; Mainen et al., 1999; Kovalchuk et al., 2000). EPSCaTs have been used to monitor the probability of transmission at individual synapses made by associational or Schaffer collateral fibers on distal apical dendrites of CA3 and CA1 pyramidal neurons (Emptage et al., 1999; Yuste et al., 1999), and we, in this way, demonstrated recently that LTP at these synapses is ex- 
pressed at least in part by presynaptic mechanisms (Emptage et al., 2003).

In previous work, we combined this optical approach with electron microscopy to demonstrate that the complex dendritic spines (also known as "thorny excrescences") at mossy fiber synapses act as discrete functional units (Reid et al., 2001). Mossy fiber boutons display multiple active zones (potential transmitter release sites), each opposite its own postsynaptic density on the complex CA3 spines (Chicurel and Harris, 1992). Here, by measuring the probability of obtaining an $\operatorname{EPSCaT}\left(p_{\mathrm{Ca}}\right)$ at individual complex spines, we estimate the aggregate $p_{r}\left(p_{s}\right.$, the probability that a release event has occurred at any or all release sites) at MF synapses on CA3 pyramidal cells. We find that MF LTP results in a robust increase in $p_{\mathrm{Ca}}$ and in the amplitude of the EPSCaTs that occur. By examining the frequency histograms of EPSCaT amplitudes before and $30 \mathrm{~min}$ after LTP induction, we conclude that potentiation at individual MF synapses results variously from an increase in $p_{r}$ at functional presynaptic release sites, from the recruitment of additional release sites, or from both, all leading to an increase in quantal content. Additionally, we have been able for the first time to visualize the "unsilencing" of previously silent synapses after the induction of LTP. This unsilencing of MF synapses appears to occur via presynaptic mechanisms (Voronin, 1994), not via addition of AMPA-type glutamate receptors at spines initially expressing only NMDA-type receptors (Isaac et al., 1995; Liao et al., 1995).

\section{Materials and Methods}

Hippocampal slices. Transverse $300 \mu \mathrm{m}$ slices of hippocampus were cut from 8-d-old male Wistar rat pups and cultured according to published methods (Yamamoto et al., 1989; Stoppini et al., 1991) on Millicell CM membranes (Millipore, Bedford, MA) for 12-28 d before use, as described previously (Emptage et al., 1999) but with the addition of B27 supplement (Invitrogen, Gaithersburg, MD) to the culture medium.

Electrophysiological and optical recording and analysis. Cultures on their supporting membranes were transferred to a recording chamber where they were continually superfused with oxygenated $\left(95 \% \mathrm{O}_{2}-5 \%\right.$ $\mathrm{CO}_{2}$ ) artificial CSF (ACSF) maintained at $30-32^{\circ} \mathrm{C}$ (temperature controller; Scientific Systems Design, Montclair, NJ). Except when noted, the ACSF contained the following (in $\mathrm{mm}$ ): $120 \mathrm{NaCl}, 3 \mathrm{KCl}, 2 \mathrm{MgSO}_{4}, 3$ $\mathrm{CaCl}_{2}, 1.2 \mathrm{NaHPO}_{4}, 23 \mathrm{NaHCO}_{3}, 11$ glucose, and 1 Trolox antioxidant. Slices were viewed through an upright microscope using a $60 \times$ numerical aperture 0.9 water immersion objective (Olympus Optical, Tokyo, Japan) and a Bio-Rad (Hercules, CA) MRC1000 or Radiance 2000 confocal laser scan head. Pyramidal cells in the CA3 region were impaled with sharp microelectrodes (100-180 M $\Omega$, with filament) tip filled with 0.5 mм Oregon Green 488 BAPTA-1 (Molecular Probes, Eugene, OR) in $200 \mathrm{~mm}$ potassium acetate and backfilled with $4 \mathrm{~m}$ potassium acetate. The indicator was injected into the cell by applying hyperpolarizing current (0.05-0.1 nA) for 5-20 min via an Axoclamp 2B amplifier (Axon Instruments, Foster City, CA); subsequent leakage from the microelectrode contributed to an additional slow increase in indicator concentration over time. Stimuli (single $40-100 \mu$ sec square pulses or pairs separated by $70 \mathrm{msec}$; stimulus amplitude was adjusted at the beginning of each experiment to $\sim 50 \%$ of threshold for eliciting action potentials to a single stimulus and maintained at that level throughout) were delivered via a sharpened monopolar tungsten electrode (A-M Systems, Carlsborg, WA) placed in the cell body layer of the dentate gyrus. The proximal region of the apical dendrite of the CA3 pyramidal neuron was searched while stimulating at $\sim 0.05 \mathrm{~Hz}$, until a spine exhibiting stimulus-evoked calcium transients was found. Two-dimensional confocal scans lacked the temporal resolution required for accurate measurement of calcium transients, so we used the confocal microscope in "line scan" mode. Line scans (ranging from 150 to 256 successive sweeps, at $2 \mathrm{msec}$ intervals) were obtained along a line passing through the spine of interest and either nearby spines or the adjacent dendritic shaft. The orientation of the line was optimized, and the precise time of stimulation was marked in the image, by either software (Bio-Rad) or hardware scan rotator and time stamp (Scientific Systems Design). LTP was induced by delivery of three $1 \mathrm{sec}$ trains of $100 \mathrm{~Hz}$ stimuli at $20 \mathrm{sec}$ intervals via the stimulating electrode. Electrophysiological data were captured using A/DVANCE (McKeller Designs, Vancouver, British Columbia, Canada) and Axograph (Axon Instruments) software, and images were collected using COMOS and LaserSharp software (Bio-Rad). Electrophysiological data and images were analyzed using Axograph and NIH Image (http://rsb. info.nih.gov/nih-image/) software, respectively. The calcium transient amplitudes were expressed as percentage fractional change in fluorescence $(\Delta F / F)$, calculated as $100\left(F-F_{\text {initial }}\right) /\left(F_{\text {initial }}-F_{\text {background }}\right)$, where $F_{\text {initial }}$ is the mean fluorescence intensity of the imaged region during a period just before stimulation. To improve the signal-to-noise ratio, we measured $\Delta F / F$ over a $30 \mathrm{msec}$ window at the peak of the $\mathrm{Ca}^{2+}$ transient. Fluorescence signals were essentially linear over the range of responses investigated here (see Fig. $1 D$ ). Events were counted as successes if amplitudes exceeded the unstimulated noise distribution, a threshold that was typically $\Delta F / F>20 \%$. $p_{\mathrm{Ca}}$ was calculated on the basis of $9-140$ trials (average, $44.4 \pm 5.0$ ) under each condition, using only the response to the first of paired stimuli. Statistical comparisons were made with Student's $t$ test, two-tailed except when indicated, or Kolmogorov-Smirnov comparison. CNQX and D-APV were obtained from Tocris Cookson (Ballwin, MO), and Trolox was from Aldrich (Milwaukee, WI); all other reagents were obtained from Sigma (St. Louis, MO).

\section{Results \\ EPSCaTs detect transmitter release at single mossy fiber synapses}

Individual pyramidal cells in the CA3 region of organotypic hippocampal slice cultures were filled with the high-affinity fluorescent $\mathrm{Ca}^{2+}$ indicator Oregon Green 488 BAPTA-1 and imaged by laser scanning confocal microscopy (Fig. $1 A$ ). Stimulation in the dentate granule cell layer at an intensity that evoked subthreshold EPSPs in the dye-filled CA3 pyramidal neuron typically produced a rapid increase in $\mathrm{Ca}^{2+}$ indicator fluorescence in one or a few complex dendritic spines, as illustrated in Figure $1 \mathrm{~A}$. We previously used a range of independent criteria, including pharmacological and ultrastructural methods, to confirm that the spines under investigation are postsynaptic to mossy fiber terminals (Reid et al., 2001). Line scans passing through these spines demonstrated a rapid stimulus-evoked increase in fluorescence with short latency, rapid rise, and slower decay (Fig. 1A). The block of EPSCaTs at these complex spines by the broad-spectrum AMPA/ kainate receptor antagonist CNQX confirmed that EPSCaTs are generated by glutamate released from presynaptic boutons (Reid et al., 2001). EPSCaTs were not invariably evoked by every stimulus (Fig. 1A). A frequency histogram constructed from 73 consecutive responses in one complex spine (Fig. $1 B, C$ ) illustrates the unambiguous resolution of EPSCaTs and failures, allowing an accurate measure of $p_{\mathrm{Ca}}$, the probability of a stimulus evoking an EPSCaT, at the mossy fiber synapse.

\section{$p_{\mathrm{Ca}}$ reflects the probability of glutamate release at mossy fiber synapses}

Mossy fiber boutons display multiple discrete active zones (potential transmitter release sites) opposite postsynaptic densities on CA3 complex spines (Chicurel and Harris, 1992). Thus, the effective $p_{r}$ at these synapses is an aggregate multinomial probability $p_{s}$, reflecting the individual $p_{r}$ at each release site and the total number of these sites.

We performed several experiments to establish whether $p_{\mathrm{Ca}}$ provided a valid estimate of $p_{s}$. Rather than reflecting failure to release transmitter, EPSCaT failures could result from nerve stimulation failure. To test this, we measured $p_{\mathrm{Ca}}$ with a random 
sequence of varying stimulus strengths (Fig. 2A). In all cases, $p_{\mathrm{Ca}}$ increased abruptly from zero to a stable plateau value (range, 0.7-1) as stimulus strength increased, indicating that, at stimulus strengths in the plateau region, failure to evoke action potentials does not contribute to $p_{\mathrm{Ca}}$. (In each experiment, stimulus strength was routinely adjusted to the stable plateau range.) Paired-pulse facilitation is believed to reflect an increase in $p_{r}$ to the second stimulus when effects of residual $\mathrm{Ca}^{2+}$ from the first impulse augment release attributable to $\mathrm{Ca}^{2+}$ from the second (Wu and Saggau, 1994; Zucker, 1999; Dittman et al., 2000; Emptage et al., 2001). When $p_{\mathrm{Ca}}$ to single stimuli is $<1$, application of paired stimuli yields a higher $p_{\mathrm{Ca}}$ to the second stimulus $(0.57 \pm$ 0.08 vs $0.88 \pm 0.07 \% ; n=10 ; p<0.05)$ (Fig. 2B) (Reid et al., 2001). Paired-pulse facilitation of $p_{\mathrm{Ca}}$ thus is consistent with the hypothesis that $p_{\mathrm{Ca}}$ is a reflection of composite $p_{r}$ at individual release sites. Finally, we examined the effects on $p_{\mathrm{Ca}}$ of adenosine $(0.5-1 \quad \mu \mathrm{M})$, a compound known to reduce transmitter release via a presynaptic mechanism (Yamamoto et al., 1993). Adenosine significantly reduced both the EPSP (from $5.9 \pm 0.7 \mathrm{mV}$ in ACSF to $2.4 \pm 0.6 \mathrm{mV}$ in adenosine; $n=6$; $p<0.01$ ) and $p_{\mathrm{Ca}}$ (from $0.95 \pm 0.03$ to $0.45 \pm 0.16 \mathrm{mV} ; n=6 ; p<0.02$ ) (Fig. $2 C$ ), a result consistent with $p_{\mathrm{Ca}}$ reflecting the aggregate probability of transmitter release at mossy fiber terminals. There was also a significant and reversible effect of adenosine on meanEPSCaT amplitude $(\Delta F / F=84 \pm 23$ vs $57 \pm 20 \% ; n=6 ; p<$ $0.05)$; however, the reduction in $p_{\mathrm{Ca}}$ was also observed at two spines in which there was no change inEPSCaT amplitude, demonstrating that the reduction in $p_{\mathrm{Ca}}$ was not a consequence of a reduction in signal. The reduction in $p_{\mathrm{Ca}}$ is therefore consistent with a presynaptic effect of adenosine. The reason for the reduction of the EPSCaT amplitude by adenosine is considered below. Bicuculline, a $\mathrm{GABA}_{\mathrm{A}}$ receptor antagonist, had no effect on $p_{\mathrm{Ca}}(0.75 \pm 0.09$ vs $0.79 \pm 0.10$ before drug; $0.5-1 \mu \mathrm{M} ; n=4$; NS), indicating that the latter is not influenced by $\mathrm{GABA}_{\mathrm{A}}$ mediated inhibitory transmission. Together, these results strongly suggest that $p_{\mathrm{Ca}}$ at the mossy fiber synapse reflects the aggregate probability of transmitter release from all functional release sites at the synapse.

\section{EPSCaT amplitudes reflect EPSP amplitudes at mossy fiber synapses}

In contrast to associational-commissural synapses, in which calcium-induced calcium release (CICR) from internal stores within the spine is a major source of the evoked postsynaptic $\mathrm{Ca}^{2+}$ transient (Emptage et al., 1999; Kovalchuk et al., 2000), CICR does not contribute toEPSCaTs evoked by single stimuli delivered to mossy fibers. Instead, theseEPSCaTs result from
$\mathrm{Ca}^{2+}$ influx through voltage-activated calcium channels (VACCs) and NMDA receptor channels, both voltage-dependent processes (Reid et al., 2001). To investigate the relationship between EPSCaT amplitudes and synaptically-evoked depolarization, we observed the effect of CNQX at subsaturating concentrations $(0.5-1.5 \mu \mathrm{M})$. CNQX at these concentrations reduced both EPSP amplitude (from $8.7 \pm 1.8$ to $4.4 \pm 1.5 \mathrm{mV} ; n=7 ; p<$ $0.005)$ and EPSCaT amplitude $(\Delta F / F$ reduced from $121 \pm 20$ to $71.6 \pm 7.5 \% ; n=10 ; p<0.01$ ) (Fig. $3 A$ ) but had no effect on $p_{\mathrm{Ca}}$ $(0.95 \pm 0.03$ vs $0.96 \pm 0.03 ; n=10)$ (Fig. $3 B)$, indicating that CNQX has purely postsynaptic effects at this synapse. Changes in EPSCaT amplitude brought about by different concentrations of CNQX are well correlated with the resulting changes in EPSP amplitude (linear regression slope, $0.75 ; r=0.71 ; p<0.03$ ) (Fig. $3 C$ ), indicating that changes in EPSCaT amplitude are useful indicators of changes in EPSP amplitude. EPSCaT amplitudes at MF synapses in response to single stimuli were not seriously compromised by indicator saturation, because larger-amplitude tran- 

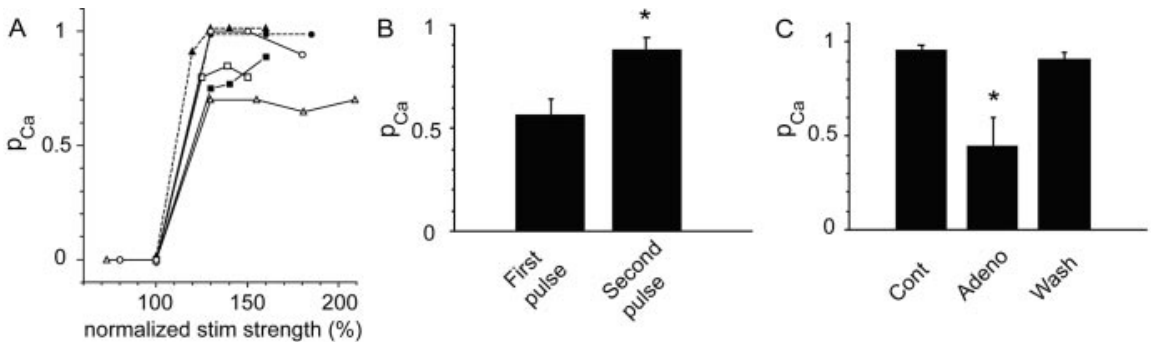

Figure 2. $\quad p_{\mathrm{Ca}}$ reflects the probability of glutamate release at mossy fiber synapses. $A, A$ plot of $p_{\mathrm{Ca}_{\mathrm{a}}}$ versus stimulus strength (normalized to the threshold for evoking an EPSCaT) demonstrates in six experiments that $p_{C_{a}}$ increases abruptly from zero to a plateau value, indicating that $p_{\mathrm{Ca}}$ is not simply a reflection of the failure to evoke action potentials in the mossy fiber axon. $B$, Summary histogram of the mean $p_{C a}$ in response to the first and second pulse of a paired-pulse stimulus paradigm (70 msec interval; $n=10)$. The paired-pulse facilitation of $p_{C_{a}}$ is consistent with $p_{C_{a}}$ reflecting $p_{s}$. C, Summary histogram of the mean $p_{C_{a}}$ before (control; Cont), during (adenosine; Aden), and after (Wash) application of adenosine $(0.5-1 \mu \mathrm{m} ; n=6)$. Adenosine, which reduces the aggregate probability of transmitter release $\left(p_{s}\right)$ from presynaptic terminals, also reduces $p_{\mathrm{Ca}}$.
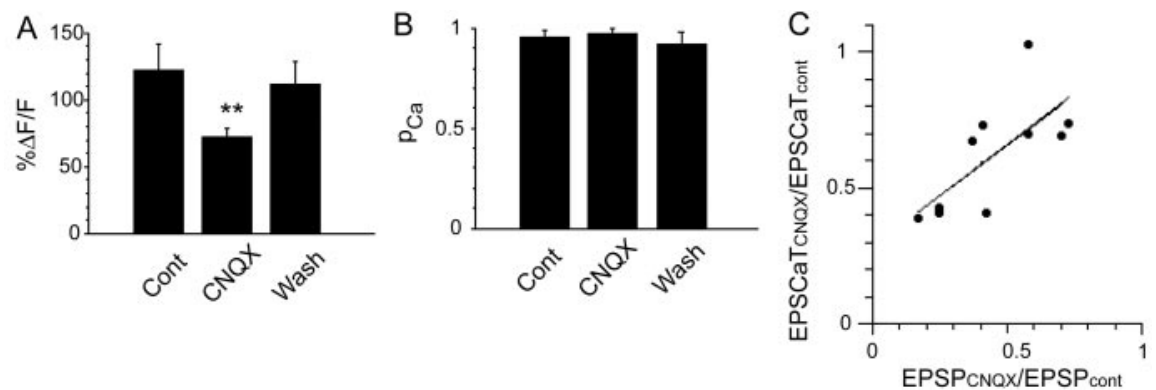

Figure 3. EPSCaT amplitudes reflect EPSP amplitudes at mossy fiber synapses. $A$, Summary histogram of mean EPSCaT amplitude before, during application, and after washout of subsaturating concentrations of the AMPA-type glutamate receptor antagonist CNQX (0.5-1.5 $\mu \mathrm{m} ; n=10)$. ${ }^{* *}$ indicate significant difference from control (before application, $\left.p<0.01\right)$; error bars represent SEM. $B$, Summary histogram of mean $p_{C_{a}}$ in the same experiments before, during application, and after washout of CNQX; there is no change in $p_{\mathrm{Ca}_{2}}$, indicating that CNQX acts only postsynaptically. C, The reduction in EPSCaT amplitude (expressed as EPSCaT CNQX $_{\text {IEPSCaT }}$ Cont, the ratio of EPSCaT amplitude in CNQX to baseline EPSCaT amplitude in ACSF) plotted against the reduction in EPSP amplitude (expressed analogously) at varying concentrations of CNQX for the 10 experiments summarized in $A$ and $B$. The changes in amplitudes are well correlated (linear regression slope, $0.75 ; r=0.71$ ), indicating that changes in EPSCaT amplitude are useful indicators of changes in EPSP amplitude.

sients were always obtained in response to backpropagating action potentials or trains of stimuli (Fig. 1D) (Reid et al., 2001).

\section{Presynaptic mechanisms can influence EPSCaT amplitude}

The magnitude of synaptically-evoked depolarization at the complex spine will depend on the number of transmitter quanta released from the multiple active zones of the mossy fiber bouton onto their discrete postsynaptic densities. Thus, the EPSCaT amplitude, which is proportional to spine depolarization (Fig. $3 C$ ), should be increased under circumstances in which $p_{s}$ is increased. Indeed, in paired-pulse experiments at synapses with $p_{\mathrm{Ca}}<1$, the amplitude of the EPSCaT to second pulses when the first pulse yielded a failure was significantly larger than the EPSCaT generated by successful first pulses ( $\Delta F / F$ second pulse, $103.8 \pm 14 \%$ vs $\Delta F / F$ first pulse, $90 \pm 7.5 \% ; n=10 ; p<0.05)$. Also, adenosine significantly reduced mean EPSCaT amplitudes even when failures are excluded (see above) (Fig. 2C), consistent with a reduction in the mean number of release events per impulse at the imaged synapses. These results demonstrate that presynaptic mechanisms can influence the EPSCaT amplitude.

\section{Optical quantal analysis of LTP at the mossy fiber synapse}

We next investigated whether the optical parameters $p_{\mathrm{Ca}}$ and EPSCaT amplitude were changed after the induction of mossy fiber LTP. An example of such an experiment is shown in Figure
4. Overall, three $1 \mathrm{sec}$ trains of $100 \mathrm{~Hz}$ stimuli at $20 \mathrm{sec}$ intervals induced robust mossy fiber LTP $(>50 \%$ increase in EPSP) in 19 of 34 cells [mean EPSP amplitude 30-40 min after induction was $195 \pm$ $8.8 \%$ of baseline $(100 \%) ; p<0.001$ ] (Fig. $5 D)$. The other 15 cells, in which the induction protocol did not increase the synaptic amplitude (EPSP amplitude $30-40$ min after induction protocol, $97.8 \pm 4.3 \%$ of baseline; $p=0.53$; NS) constituted a tetanized but nonpotentiated control group. In a separate group of experiments without imaging, MF LTP was induced by the same stimulus protocol in the presence of APV (EPSP amplitude after tetanus, $237 \pm 28 \%$ of baseline; $n=3$ ), confirming that LTP induced by this protocol was independent of NMDA receptor activation. [APV was not routinely included in the superfusate in imaging experiments because it reduced the EPSCaT amplitude (Reid et al., 2001).] The LTP induction protocol had minimal effect $(1.1 \pm 0.9 \mathrm{mV}$ depolarization) on the resting membrane potential.

In potentiated synapses in which $p_{\mathrm{Ca}}$ at the imaged complex spine was initially $<1$, $p_{\mathrm{Ca}}$ was significantly increased after LTP induction $(0.50 \pm 0.08$ vs $0.85 \pm 0.03 ; p<$ $0.005 ; n=19$ ) (Fig. $5 A$ ). (The few synapses with initial $p_{\mathrm{Ca}}=1$ were excluded from this analysis, because they could not manifest any increase in $p_{\mathrm{Ca}}$.) In control synapses with initial $p_{\mathrm{Ca}}<1$ in which stimulation did not yield LTP, $p_{\mathrm{Ca}}$ was unaltered $(0.58 \pm 0.10$ vs $0.55 \pm 0.11$; NS; $n=12)$ (Fig. $5 A$ ). Comparison of the change in $p_{\mathrm{Ca}}$ (post - pre) confirmed a significant difference between potentiated and control populations $(0.34 \pm$ $0.08, n=19$ vs $-0.01 \pm 0.03, n=12 ; p<0.005)$ (Fig. $5 B$ ). Overall in the potentiated group, there was also a nonsignificant increase in the EPSCaT amplitude $(\Delta F / F=104.7 \pm 11.3 \%$ pretetanus vs $113.2 \pm 11.4 \%$ post-tetanus; $p=0.16$ ). In contrast, there was a small reduction in the EPSCaT amplitude in the tetanized but nonpotentiated control group $(\Delta F / F=110 \pm 17.1$ vs $93.0 \pm 12.0 \% ; p<0.06$ ), a consequence of the slow leak of additional indicator from the recording pipette that occurred equally in both groups (baseline fluorescence, $F_{\text {initial }}$, increased $75.4 \pm$ $16.7 \%$ in the potentiated group and $77.5 \pm 47.1 \%$ in the control group). A comparison of changes in EPSCaT amplitude in potentiated versus nonpotentiated groups showed a significant difference (ratio of $\% \Delta F / F$ post-tetanus to $\% \Delta F / F$ pre-tetanus, $1.20 \pm$ 0.11 vs $0.91 \pm 0.08 ; p<0.05$ ) (Fig. $5 C$ ). These results provide additional evidence that LTP is associated with an increased EPSCaT, and hence EPSP amplitude, at the individual potentiated synapse.

LTP-associated increases in EPSCaT and EPSP amplitude could result from postsynaptic mechanisms (e.g., changes in the number and/or kinetic properties of functional postsynaptic AMPA receptors, VACCs, or NMDA receptors) or from presynaptic mechanisms that increase quantal content. Such mechanisms cannot be distinguished solely by comparison of mean 
A

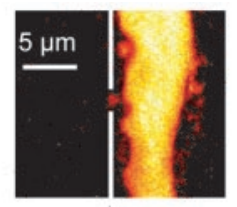

$\uparrow$
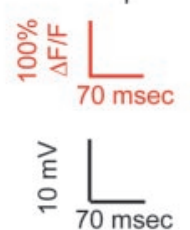

B

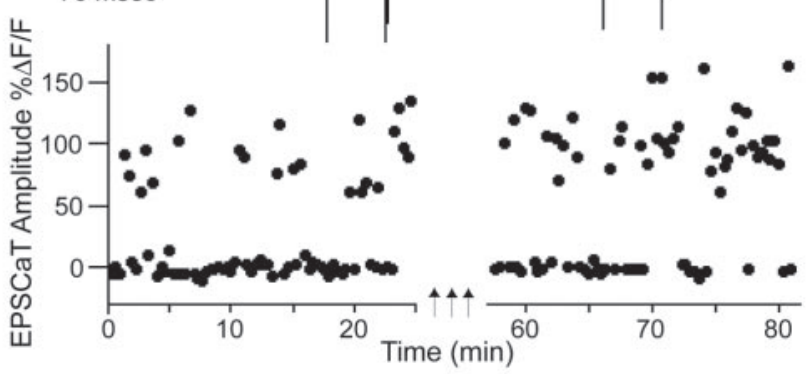

C

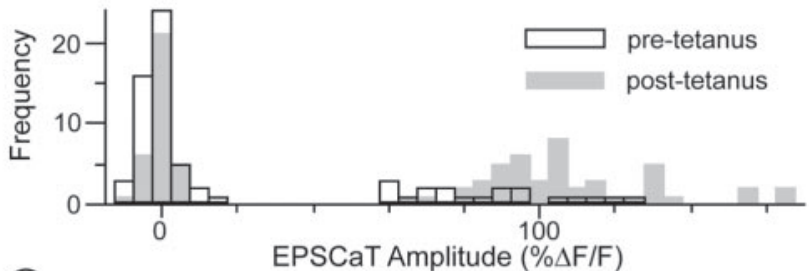

D

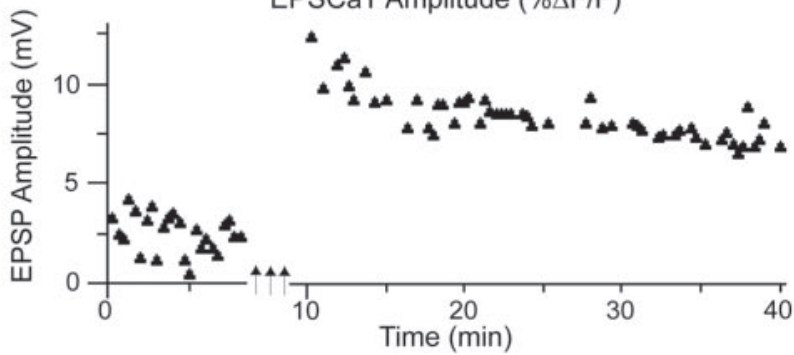

Figure 4. Mossy fiber LTP results in an increase in $p_{\mathrm{Ca}}$ and EPSCaT amplitude. $A$, Left, Proximal apical dendritic segment of a CA3 pyramidal neuron as in Figure 1. The soma is above the FOV; the apical dendrite extends distally downward. The middle and right panels show representative line scans through a large spine (in gap in white line in the left panel) in response to dentate granule cell layer paired-pulse stimulation, before (pre-tetanus, middle panel) and 30 min after (post-tetanus, right panel) induction of LTP by three 1 sec trains of stimulation at 100 $\mathrm{Hz}$ separated by $20 \mathrm{sec}$. (Traces show spine calcium signals and simultaneous intracellular voltage recordings as in Fig. 1.) In this experiment, mean EPSP amplitude 30 min after LTP induction had increased to $246 \%$ of baseline $(D) ; p_{C_{a}}$ increased from 0.27 before LTP induction to 0.58 afterward $(B)$, whereas average EPSCaT amplitude (successes only) increased from $\Delta F / F=86$ to $108 \%$ (C).

group data; therefore, we next analyzed EPSCaT amplitude frequency histograms in individual experiments.

EPSCaT amplitude frequency histograms indicate that increases in both $p_{\mathrm{r}}$ and $N$ can contribute to mossy fiber LTP By examining EPSCaT amplitude frequency histograms from individual MF synapses, we can obtain information about how quantal parameters at specific synapses are altered in LTP. Figure 6 shows examples of EPSCaT amplitude frequency histograms obtained before and beginning $30 \mathrm{~min}$ after the induction of LTP at four different MF synapses. Extensive sampling in these experiments was limited by the need to avoid photo damage, and it was
A
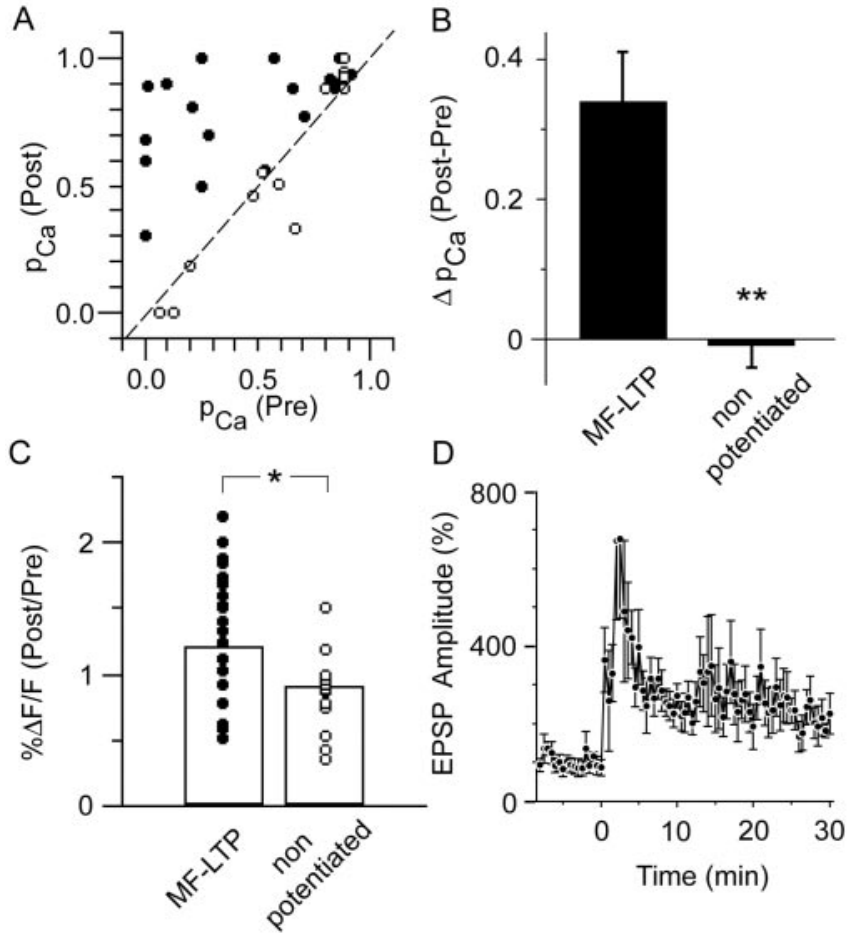

Figure 5. Changes in optical quantal parameters of transmission at single mossy fiber synapses after the induction of LTP. A, Plot of $p_{\mathrm{Ca}_{\mathrm{a}}} 30-45 \mathrm{~min}$ after LTP induction versus $p_{\mathrm{Ca}_{\mathrm{a}}}$ before LTP induction, demonstrating that the probability of a stimulus evoking an EPSCaT was increased in nearly all responding spines in potentiated preparations (filled circles) but not in the tetanized but nonpotentiated control group sampled at corresponding intervals (open circles). Dashed line at $45^{\circ}$ represents the null hypothesis of no change in $p_{\mathrm{Ca}}$. B, Summary histogram showing the data of $(a)$ plotted as change in $p_{C_{a}}$, confirming that $p_{C_{a}}$ is significantly increased after LTP induction ( $\left.{ }^{*} p<0.005\right)$. C, Summary histogram plotting the ratio of EPSCaT amplitudes 30 - 45 min after LTP induction/before induction (filled circles) compared with the tetanized but nonpotentiated control group (open circles) sampled at corresponding time points. The mean ratio in the potentiated group is $>1$ and is significantly greater than the mean ratio in the nonpotentiated control group $\left(^{*}\right)$, which is $<1$. Thus, both $p_{\mathrm{Ca}}$ and EPSCaT amplitude at individual mossy fiber synapses are increased after LTP induction. $D$, Time course of the mean EPSP amplitude before and after LTP induction in the potentiated group, expressed as percentage of baseline amplitude ( $n=6$; those cells in which stimulation after LTP frequently evoked spiking are not included here). Error bars in $B$ and $D$ indicate SEM.

in general not possible to fit Gaussian functions to the data. Nevertheless, several qualitatively different patterns of changes in the histograms emerged at the potentiated, but never in the unpotentiated control, synapses. The experiments selected for individual analysis showed robust LTP and no substantial rundown of the EPSCaT amplitude during either the pre-LTP or post-LTP recording epochs.

At the synapse in Figure 6A, LTP was associated with a clear increase in $p_{\mathrm{Ca}}$ (from 0.82 pre to 0.98 post; $p<0.02$; MannWhitney comparison), but there was no significant change in the distribution of EPSCaT amplitudes (mean $\Delta F / F$ for non-failures, $177.4 \pm 3.7 \%$ pre vs $167 \pm 2.7 \%$ post; NS; Kolmogorov-Smirnov comparison). Although the breadth of these amplitude distributions (presumably a consequence of multiplication of EPSP variance by variance in calcium channel activation and by small variations in plane of focus between successive imaging sessions) makes it difficult to exclude other explanations, this result is most simply accounted for by an increase in $p_{r}$ at an active release site: given the high initial $p_{\mathrm{Ca}}$ of the response, any recruitment of additional release sites would have been likely to result in multiple-release events with larger EPSCaT amplitudes. Similar 
A

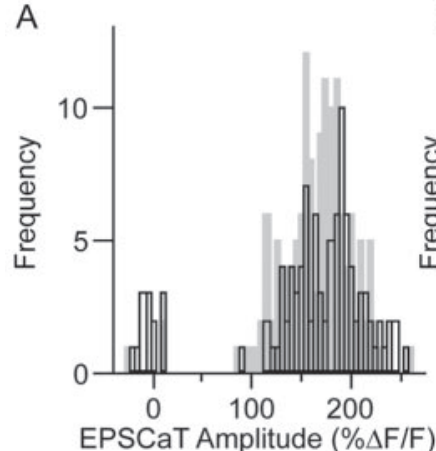

B
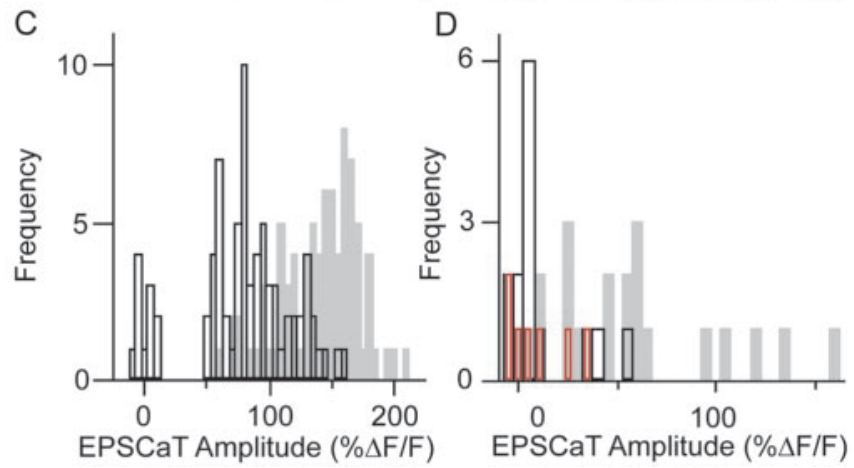

Figure 6. Frequency histograms of EPSCaT amplitudes indicate that increases in both $p_{r}$ and in the number of active release sites can contribute to the tetanus-induced increase in efficacy of individual mossy fiber synapses. $A$, Amplitude frequency histogram of EPSCaTs recorded at a single mossy fiber synapse before (white bars) and 30 min after (gray bars) LTP induction. In this experiment, induction led to an increase in $p_{\mathrm{Ca}}$ (note reduction in failures), with no change in the apparently unimodal distribution of EPSCaT amplitudes. This type of change, also observed at six other synapses in independent experiments, suggests that an increase in efficacy at the observed synapse resulted from a simple increase in the probability of transmitter release. $B$, Amplitude frequency histogram at another mossy fiber synapse. In this case, there were no failures, and EPSCaT amplitudes clustered unimodally around $\Delta F / F=50 \%$ before LTP induction (white bars). After LTP induction (gray bars), most EPSCaTs had substantially larger amplitudes; a similar emergence of new, high-amplitude responses after LTP was observed at three other synapses in independent experiments and is most easily explained by the recruitment of at least one additional active release site. C, At this MF synapse, LTP induction resulted in both an increase in $p_{\mathrm{Ca}}$ and the emergence of higher-amplitude EPSCaTs. D, An additional example of an MF synapse at which LTP induction resulted in both an increase in $p_{\mathrm{Ca}_{\mathrm{a}}}$ and the emergence of higher-amplitude EPSCaTs. Addition of adenosine to the superfusate after the collection of post-LTP induction data resulted in a reduction of $p_{\mathrm{Ca}}$ and elimination of the higher-amplitude EPSCaTs (red bars), to values similar to those seen before potentiation. The pattern of change illustrated in C and D was observed at six other synapses in independent experiments and is also consistent with an LTP-associated recruitment of additional active release sites.

LTP-related changes in EPSCaT amplitude distribution were obtained in six other experiments. Thus, at least in some cases, MF LTP appears to be attributable to a simple increase in $p_{r}$ at an existing functional release site.

A second type of change in the EPSCaT amplitude frequency histogram after the induction of LTP was the addition of largeramplitude EPSCaTs. Such a pattern without a change in $p_{\mathrm{Ca}}$ is illustrated in Figure $6 B$. Here, before LTP induction, $p_{\mathrm{Ca}}$ was 1 , and EPSCaT amplitudes were unimodally distributed with mean $\Delta F / F=49.5 \pm 2.0 \%$, a pattern consistent with a single functional release site with $p_{r}=1$. After LTP induction, the mean EPSCaT amplitude $(\Delta F / F)$ was increased to $80.8 \pm 6.3 \%$, with values ranging up to $>130 \%$ but also including events with amplitudes in the pre-LTP range. Similar LTP-related changes in EPSCaT amplitude distribution but not $p_{\mathrm{Ca}}$ were obtained in three other experiments. An increase in quantal amplitude at the previously functional release site could not be solely responsible for this type of change, because this would have resulted in a right-shifted unimodal distribution. The emergence of multiquantal release at the original release site cannot be excluded but would be an unlikely explanation of the change if, as has been reported, release of a single quantum of transmitter leads to occupation of most postsynaptic receptors opposite the release site (Jonas et al., 1993). Instead, this pattern is most easily explained by an increase in $N$ through the recruitment of one or more additional release sites with relatively high $p_{r}$, activated independently of the initial site. In either case, large-amplitude events presumably represent multivesicular release, either at the original release site or by simultaneous activation of the original and newly recruited release sites, whereas the smallest-amplitude events represent univesicular release.

Variants of this second type of change after LTP are illustrated in Figure $6, C$ and $D$. At the synapse in Figure $6 C, p_{\mathrm{Ca}}$ was initially 0.86 , with EPSCaTs having mean amplitude $\Delta F / F=95.1 \pm 3.4 \%$. After LTP induction, $p_{\mathrm{Ca}}$ increased to 1 , and the EPSCaT amplitude distribution (mean $\Delta F / F=145.7 \pm 3.3 \%$ ) was significantly altered ( $p<0.0001$; Kolmogorov-Smirnov comparison), ranging from preinduction amplitudes to substantially larger amplitudes. At the synapse in Figure $6 D, p_{\mathrm{Ca}}$ was initially low $(0.21)$, with the rare EPSCaTs having mean amplitude $\Delta F / F=43.7 \pm$ $6.6 \%$; after LTP induction, $p_{\mathrm{Ca}}$ increased to 0.81 , with EPSCaTs (mean $\Delta F / F=76.7 \pm 10.0 \%$ ) again ranging from preinduction amplitudes to substantially larger amplitudes. Similar patterns, of change in both $p_{\mathrm{Ca}}$ and EPSCaT amplitude distribution after LTP induction, were observed at six other synapses and are most simply explained by the activation of new synaptic junctions in these complex synapses, presumably by recruitment of additional release sites at an initially single-release-site bouton. In the experiment of Figure $6 D$, adenosine was added to the perfusate after the completion of post-LTP sampling, resulting in a reduction of both $p_{\mathrm{Ca}}$ and EPSCaT amplitudes to levels similar to those before LTP induction, reinforcing the interpretations above. Similar changes in the EPSCaT amplitude histograms during application of adenosine were observed at two additional MF synapses.

Figure 7 illustrates a third type of change in the frequency distribution of EPSCaT amplitudes seen after the induction of LTP: the activation of silent synapses. Before the induction of LTP, no EPSCaTs could be evoked at this synapse, even by paired (70 msec interval) stimuli. After LTP induction, however, stimulation elicited responses with high $p_{\mathrm{Ca}}$ and with a broad range of amplitudes. Similar unsilencing after LTP induction was observed in three other experiments. In two experiments, preparations were exposed to $0 \mathrm{Mg}^{2+}$ ACSF for a 15 min period before LTP induction; this manipulation failed to reveal EPSCaTs at the synapses subsequently unsilenced by LTP.

In summary, at 19 synapses studied before and after LTP induction, our optical quantal analyses revealed two modes of expression of the potentiation. In the first, LTP was associated with a reduction in failure rate (i.e., an increase in $p_{\mathrm{Ca}}$ ), without significant change in distribution of successful response amplitudes $(n=7)$. In the second, LTP was associated with the emergence of new, larger-amplitude responses $(n=12$, of which 8 , in which $p_{\text {Ca }}$ was initially $<1$, were also accompanied by a reduction in failure rate). In addition, a third mode of LTP expression was detected serendipitously at four other synapses present within the field of view (FOV) of other investigated synapses: activation of previously silent synapses. 

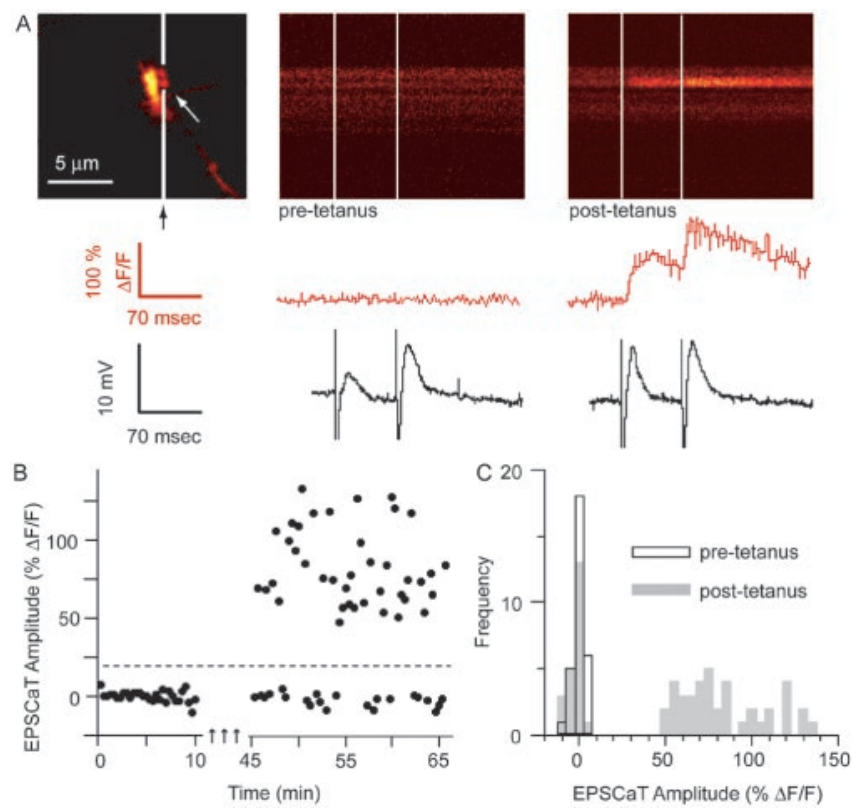

Figure 7. Induction of mossy fiber LTP can result in the activation of silent synapses. A, Left, Proximal apical dendritic segment of a CA3 pyramidal neuron as in Figures 1 and 4 . The soma is outside the focal plane of the image. The middle and right panels show representative line scans through two large spines, the lower of which (white arrow in the left panel) is initially entirely unresponsive to paired-pulse stimulation in the dentate granule cell layer (pre-tetanus, middle panel); 30 min after (post-tetanus, right panel) induction of LTP, the synapse has become active. (Red and black traces show spine calcium signals and intracellular voltage signals, respectively, as in Figs. 1 and 4.) In this experiment, mean EPSP amplitude 30 min after LTP induction had increased to $164 \%$ of baseline; $p_{\mathrm{Ca}_{\mathrm{C}}}$ increased from 0 before LTP induction to 0.57 afterward $(B)$; the EPSCaT amplitude frequency histograms before and after LTP induction are shown in C. A similar pattern of change was observed at three other synapses in independent experiments.

\section{Discussion}

The strength of a synaptic connection between two neurons reflects three quantal parameters of transmission (the number of functional release sites, $N$, at which the presynaptic neuron releases transmitter onto the postsynaptic cell; the probability of transmitter release, $p_{r}$, at each release site; and the amplitude of the response attributable to quantal release at each site, $q$ ), which may in turn reflect a variety of molecular, structural, and kinetic factors (Choi et al., 2000). Although numerous electrophysiological methods have been used to examine how activity-dependent changes in synaptic efficacy are expressed, interpretation has been complicated by uncertainty about the applicability of their theoretical assumptions and/or by the practical ambiguities of recording from an unknown number of synapses at unknown dendritic locations. Thus, for example, the simple binomial model of transmitter release (i.e., a fixed population of release sites with identical quantal parameters) used in many analyses of coefficients of variance may be inappropriate for synapses in the CNS (Faber and Korn, 1991). Other approaches (including fitting Gaussian distributions to peaky histograms, analysis of changes in the ratio of paired-pulse synaptic amplitudes, usedependent pharmacological blockade of NMDA glutamate receptors to measure $p_{r}$, analysis of glutamate transport currents, and analysis of miniature synaptic currents) have been taken in an effort to resolve the basis of LTP expression, but continuing controversy in this field, particularly in the context of LTP at hippocampal Schaffer collateral synapses (Isaac et al., 1996; Malenka and Nicoll, 1999), testifies to the difficulty in interpret- ing the results obtained. Although there is a general consensus that MF LTP is expressed presynaptically, even here there is room for disagreement, with at least one quantal analysis indicating a purely postsynaptic locus of expression for MF LTP (Yamamoto et al., 1992); in addition, a recent study describes a form of MF long-term depression in which both induction and expression are postsynaptic (Lei et al., 2003). We therefore pursued an alternative experimental approach to this question, using optical methods to determine the quantal parameters of synaptic transmission at individual active synapses before and after LTP induction (Emptage et al., 2003).

Optical quantal analysis has certain distinct advantages over ordinary electrophysiological approaches. Because all activated inputs to a cell contribute to the electrical responses recorded at the soma, quantal analysis of these electrical responses is complicated by any heterogeneity among the activated inputs. Even paired intracellular recording is not immune to this difficulty if the two cells are connected by more than one synapse. This problem is avoided by the optical measurement of quantal parameters at an individual active synapse. In addition, the morphological criteria used to identify thorny excrescences permit the simple and unambiguous identification of mossy fiber synapses.

In previous work, we found that $p_{\mathrm{Ca}}$, the probability of obtaining an EPSCaT, can be used to estimate $p_{s}$ both at simple associational-commissural synapses (Emptage et al., 1999) and at complex mossy fiber synapses (Reid et al., 2001). The present observations of paired-pulse facilitation of $p_{\mathrm{Ca}}$, and of a reduction of $p_{\mathrm{Ca}}$ in the presence of adenosine (Figs. $2 C, 6 D$ ), confirm that $p_{\mathrm{Ca}}$ measured at a complex spine reflects the aggregate $p_{s}$ of all functional release sites at the imaged synapse. We also find (Fig. 3) at MF synapses [but not at associational-commissural synapses, in which EPSCaTs result from release of calcium from internal stores within the spine (Emptage et al., 1999)] that the EPSCaT amplitude is proportional to the size of the EPSP. (Notably, subsaturating concentrations of CNQX that substantially and proportionately reduce EPSCaT and EPSP amplitudes had no effect on $p_{\mathrm{Ca}}$, indicating that observed changes in $p_{\mathrm{Ca}}$ are not artifacts of postsynaptic phenomena or crossing of detection thresholds.) However, MF synapses typically have multiple presynaptic release sites each opposed to its own postsynaptic density (Amaral and Dent, 1981; Chicurel and Harris, 1992; Reid et al., 2001), and transmission at these synapses is therefore commonly multiquantal (Jonas et al., 1993). Altered EPSCaT amplitudes can thus reflect changes in quantal content as well as in quantal amplitude. Fortunately, these alternatives can be discriminated by inspection of EPSCaT amplitude frequency histograms. We did not observe a shift in the amplitude of the first peak in any of the experiments in which LTP was induced, a result that is inconsistent with an increase in quantal amplitude. Moreover, adenosine reduces mean EPSCaT amplitudes (Fig. 2C) by selectively abolishing the larger, multiquantal, EPSCaTs (Fig. $6 D$ ) but has little effect on the smallest-amplitude, presumably uniquantal, EPSCaTs, implying that adenosine acts presynaptically to reduce quantal content without affecting quantal amplitude. Together, these experiments demonstrate the interpretability of optical quantal analyses at individual mossy fiber synapses.

The optical quantal analyses described here demonstrate that LTP at individual MF synapses is associated with a large increase in $p_{\mathrm{Ca}}$ and thus with an increase in $p_{s}$, the aggregate probability of transmitter release from the mossy fiber bouton. This increase may be attributable to increased $p_{r}$ at already functional release sites (Fig. 6A) and/or to the recruitment of additional functional release sites or transition from uniquantal to multiquantal release 
(Fig. $6 B-D$ ). The resulting increase in mean quantal content is in agreement with previous reports that the expression of MF LTP has a presynaptic locus (Staubli et al., 1990; Zalutsky and Nicoll, 1990; Xiang et al., 1994; Weisskopf and Nicoll, 1995; LopezGarcia et al., 1996; Maeda et al., 1997). Mean EPSCaT amplitudes were also consistently increased after MF LTP induction. EPSCaT amplitude frequency histograms have allowed us to identify the quantal parameters responsible for these changes. Thus, the presence, after MF LTP induction, of EPSCaTs with amplitude similar to pre-LTP values together with EPSCaTs of much larger amplitude (Fig. $6 B, C$ ) is most easily explained by the recruitment of additional functional release sites with no change in quantal amplitude. Multivesicular release at a single release site (rather than recruitment of new functional release sites) could also account for the larger post-LTP EPSCaT amplitudes, if the AMPA receptors postsynaptic to an individual release site are not saturated by release of a single vesicle. However, there is evidence that the majority, and perhaps $>80 \%$, of postsynaptic glutamate receptors opposite an MF release site are occupied at the peak of a quantal response (Jonas et al., 1993). Because the larger EPSCaTs seen after LTP induction are often more than double the pre-LTP amplitudes (Fig. 5B,C; note that indicator nonlinearity at higher $\mathrm{Ca}^{2+}$ concentrations would tend to underestimate the magnitude of large events), it is therefore unlikely that multivesicular release at previously univesicular release sites is the only source of these larger EPSCaTs.

The results illustrated in Figure 7 represent the first visualization of LTP expression through the activation of a previously silent synapse. Studies in dissociated cell cultures have suggested that LTP at dentate granule cell autapses is expressed through the switching on of previously silent release sites (Tong et al., 1996), and it has been reported that MF synapses with functional postsynaptic AMPA and NMDA glutamate receptors can appear silent because of very low probability of transmitter release (Gasparini et al., 2000). Although we cannot exclude the possibility that the unsilencing illustrated in Figure 7 represents insertion of receptors at a postsynaptically silent synapse, this seems unlikely given the failure of $0 \mathrm{Mg}^{2+}$ ACSF to reveal subsequently unsilenced synapses and the observation that complex spine membrane opposite MF active zones are always immunoreactive for AMPA receptors, in contrast to spines at simple synapses in stratum radiatum, which are often immunonegative (Nusser et al., 1998). Thus, we consider it more likely that such unsilencing results from presynaptic mechanisms similar to those illustrated in Figure 6. Because unsilencing was detected serendipitously at synapses in the same region of dendrite as initially active synapses, it may be a substantial component of the expression of MF LTP, accounting for the fact that the mean increase in EPSP amplitude, reflecting all potentiated synapses, is larger than the mean increase in EPSCaT amplitude observed at a single complex spine. We note that, if MF LTP induction is triggered by postsynaptic events (Yeckel et al., 1999; Contractor et al., 2002), presynaptic unsilencing represents a breakdown of synapse specificity of long-term plasticity (Engert and Bonhoeffer, 1997).

In summary, these results indicate that potentiation at individual MF synapses results from an increase in quantal content, attributable to an increase in $p_{r}$ at functional presynaptic release sites, an increase in multivesicular release, and/or the recruitment of new release sites, including the activation of previously silent synapses. Our results thus support a predominantly presynaptic locus of MF LTP expression. The molecular mechanisms responsible for these presynaptic effects are not known but could include alterations in presynaptic voltage-gated channels (Mellor et al., 2002) or recruitment of $\mathrm{Ca}^{2+}$ channels (Missler et al., 2003), leading to increased $\mathrm{Ca}^{2+}$ transients at release sites, as well as activation or addition of Rab3A and related proteins (Castillo et al., 1997) regulating vesicle release. Given the progressive increase in number of releases sites seen during development of MF synapses (Stirling and Bliss, 1978; Amaral and Dent, 1981), a possible mechanism of particular interest is the addition of release sites de novo, via incorporation of new active zone precursors (Zhai et al., 2001). Resolution of this important issue awaits additional study. It also remains to be determined to what extent long-term depression at MF synapses (Kobayashi et al., 1996) is manifest through similar but opposite changes in quantal parameters, as might be suggested by evidence of the involvement of cAMP and CAMP-dependent protein kinase in both processes (Tzounopoulos et al., 1998). The experiments presented here were all performed on organotypic hippocampal slice cultures; although it remains to be determined whether MF LTP is expressed by similar mechanisms in acutely prepared slices, it is reasonable to expect that basic physiological processes in these preparations are equivalent (De Simoni et al., 2003).

Finally, in keeping with our interpretation that MF LTP is expressed by exclusively presynaptic mechanisms, we note that in none of our experiments did we detect changes in morphology of the activated spine, or formation of new dendritic spines or filopodia in its vicinity, after induction of LTP. These optical analyses extended up to 60 min after LTP induction; our observations thus pertain to a relatively early stage in LTP expression. The mechanisms by which potentiation is expressed may evolve over time (Frey et al., 1993). For example, the occlusion of paired-pulse facilitation $30 \mathrm{~min}$ after MF LTP induction (Zalutsky and Nicoll, 1990), commonly taken to indicate a presynaptic locus for expression of the potentiation, decays within $3 \mathrm{hr}$, even when MF LTP persists, whereas MF LTP beyond $3 \mathrm{hr}$, but not the early phase, is blocked by inhibitors of RNA and protein synthesis (Huang et al., 1994). Thus, different mechanisms appear to underlie the sequential stages of MF LTP. It remains to be determined whether late stages of MF LTP are characterized by changes in quantal parameters similar to those described here.

\section{References}

Amaral DG, Dent JA (1981) Development of the mossy fibers of the dentate gyrus. I. A light and electron microscopic study of the mossy fibers and their expansions. J Comp Neurol 195:51-86.

Bliss TV, Collingridge GL (1993) A synaptic model of memory: long-term potentiation in the hippocampus. Nature 361:31-39.

Castillo PE, Janz R, Sudhof TC, Tzounopoulos T, Malenka RC, Nicoll RA (1997) Rab3A is essential for mossy fibre long-term potentiation in the hippocampus. Nature 388:590-593.

Chicurel ME, Harris KM (1992) Three-dimensional analysis of the structure and composition of CA3 branched dendritic spines and their synaptic relationships with mossy fiber boutons in the rat hippocampus. J Comp Neurol 325:169-182.

Choi S, Klingauf J, Tsien RW (2000) Postfusional regulation of cleft glutamate concentration during LTP at "silent synapses." Nat Neurosci 3:330-336.

Contractor A, Rogers C, Maron C, Henkemeyer M, Swanson GT, Heinemann SF (2002) Trans-synaptic Eph receptor-ephrin signaling in hippocampal mossy fiber LTP. Science 296:1864-1869.

De Simoni A, Griesinger CB, Edwards FA (2003) Development of rat CA1 neurones in acute versus organotypic slices: role of experience in synaptic morphology and activity. J Physiol (Lond) 550:135-147.

Dittman JS, Kreitzer AC, Regehr WG (2000) Interplay between facilitation, depression, and residual calcium at three presynaptic terminals. J Neurosci 20:1374-1385.

Emptage N, Bliss TVP, Fine A (1999) Single synaptic events evoke NMDA receptor mediated release of calcium from internal stores in hippocampal dendritic spines. Neuron 22:115-124. 
Emptage N, Reid CA, Fine A (2001) Calcium stores in hippocampal synaptic boutons mediate short term plasticity, store-operated $\mathrm{Ca}^{2+}$ entry and spontaneous transmitter release. Neuron 29:197-208.

Emptage NJ, Reid CA, Fine A, Bliss TVP (2003) Optical quantal analysis reveals a presynaptic component of LTP at hippocampal schafferassociational synapses. Neuron 38:797-804.

Engert F, Bonhoeffer T (1997) Synapse specificity of long-term potentiation breaks down at short distances. Nature 388:279-284.

Faber DS, Korn H (1991) Applicability of the coefficient of variation method for analyzing synaptic plasticity. Biophys J 60:1288-1294.

Frey U, Huang YY, Kandel ER (1993) Effects of cAMP simulate a late stage of LTP in hippocampal CA1 neurons. Science 260:1661-1664.

Gasparini S, Saviane C, Voronin LL, Cherubini E (2000) Silent synapses in the developing hippocampus: lack of functional AMPA receptors or low probability of glutamate release? Proc Natl Acad Sci USA 97:9741-9746.

Harris EW, Cotman CW (1986) Long-term potentiation of guinea pig mossy fiber responses is not blocked by $\mathrm{N}$-methyl-D-aspartate. Neurosci Lett 70:132-137.

Huang YY, Li XC, Kandel ER (1994) cAMP contributes to mossy fiber LTP by initiating both a covalently mediated early phase and macromolecular synthesis-dependent late phase. Cell 79:69-79.

Isaac JT, Nicoll RA, Malenka RC (1995) Evidence for silent synapses: implications for the expression of LTP. Neuron 15:427-434.

Isaac JT, Oliet SH, Hjelmstad GO, Nicoll RA, Malenka RC (1996) Expression mechanisms of long-term potentiation in the hippocampus. J Physiol (Paris) 90:299-303.

Johnston D (1992) NMDA-receptor independent LTP. Neurochem Int 20:461-462.

Jonas P, Major G, Sakmann B (1993) Quantal components of unitary EPSCs at the mossy fibre synapse on CA3 pyramidal cells of rat hippocampus. J Physiol (Lond) 472:615-663.

Kobayashi K, Manabe T, Takahashi T (1996) Presynaptic long-term depression at the hippocampal mossy fiber-CA3 synapse. Science 273:648-650.

Kovalchuk Y, Eilers J, Lisman J, Konnerth A (2000) NMDA receptormediated subthreshold $\mathrm{Ca}^{2+}$ signals in spines of hippocampal neurons. J Neurosci 20:1791-1799.

Lei S, Pelkey KA, Topolnik L, Congar P, Lacaille JC, McBain CJ (2003) Depolarization-induced long-term depression at hippocampal mossy fiber-CA3 pyramidal neuron synapses. J Neurosci 23:9786-9795.

Liao D, Hessler NA, Malinow R (1995) Activation of postsynaptically silent synapses during pairing-induced LTP in CA1 region of hippocampal slice. Nature 375:400-404.

Lopez-Garcia JC, Arancio O, Kandel ER, Baranes D (1996) A presynaptic locus for long-term potentiation of elementary synaptic transmission at mossy fiber synapses in culture. Proc Natl Acad Sci USA 93:4712-4717.

Maeda T, Kaneko S, Akaike A, Satoh M (1997) Direct evidence for increase in excitatory amino acids release during mossy fiber LTP in rat hippocampal slices as revealed by the patch sensor methods. Neurosci Lett 224:103-106.

Mainen ZF, Malinow R, Svoboda K (1999) Synaptic calcium transients in single spines indicate that NMDA receptors are not saturated. Nature 399:151-155.

Malenka RC, Nicoll RA (1999) Long-term potentiation-a decade of progress? Science 285:1870-1874.

Malinow R, Otmakhov N, Blum KI, Lisman J (1994) Visualizing hippocampal synaptic function by optical detection of $\mathrm{Ca}^{2+}$ entry through the $N$-methyl-D-aspartate channel. Proc Natl Acad Sci USA 91:8170-8174.

Mellor J, Nicoll RA (2001) Hippocampal mossy fiber LTP is independent of postsynaptic calcium. Nat Neurosci 4:125-126.

Mellor J, Nicoll RA, Schmitz D (2002) Mediation of hippocampal mossy fiber long-term potentiation by presynaptic Ih channels. Science 295:143-147.

Missler M, Zhang W, Rohlmann A, Kattenstroth G, Hammer RE, Gottmann K, Sudhof TC (2003) Alpha-neurexins couple $\mathrm{Ca}^{2+}$ channels to synaptic vesicle exocytosis. Nature 424:939-948.

Muller W, Connor JA (1991) Dendritic spines as individual neuronal compartments for synaptic $\mathrm{Ca}^{2+}$ responses. Nature 354:73-76.

Nusser Z, Lujan R, Laube G, Roberts JD, Molnar E, Somogyi P (1998) Cell type and pathway dependence of synaptic AMPA receptor number and variability in the hippocampus. Neuron 21:545-559.

Reid CA, Fabian-Fine R, Fine A (2001) Postsynaptic calcium transients evoked by activation of individual hippocampal mossy fiber synapses. J Neurosci 21:2206-2214.

Staubli U, Larson J, Lynch G (1990) Mossy fiber potentiation and long-term potentiation involve different expression mechanisms. Synapse 5:333-335.

Stirling RV, Bliss TVP (1978) Mossy fibre development at the ultrastructural level. Prog Brain Res 48:191-198.

Stoppini L, Buchs PA, Muller D (1991) A simple method for organotypic cultures of nervous tissue. J Neurosci Methods 37:173-182.

Tong G, Malenka RC, Nicoll RA (1996) Long-term potentiation in cultures of single hippocampal granule cells: a presynaptic form of plasticity. Neuron 16:1147-1157.

Tzounopoulos T, Janz R, Sudhof TC, Nicoll RA, Malenka RC (1998) A role for cAMP in long-term depression at hippocampal mossy fiber synapses. Neuron 21:837-845.

Voronin LL (1994) Quantal analysis of hippocampal long-term potentiation. Rev Neurosci 5:141-170.

Weisskopf MG, Nicoll RA (1995) Presynaptic changes during mossy fibre LTP revealed by NMDA receptor-mediated synaptic responses. Nature 376:256-259.

Williams S, Johnston D (1989) Long-term potentiation of hippocampal mossy fiber synapses is blocked by postsynaptic injection of calcium chelators. Neuron 3:583-588.

Wu LG, Saggau P (1994) Presynaptic calcium is increased during normal synaptic transmission and paired-pulse facilitation, but not in long-term potentiation in area CA1 of hippocampus. J Neurosci 14:645-654.

Xiang Z, Greenwood AC, Kairiss EW, Brown TH (1994) Quantal mechanism of long-term potentiation in hippocampal mossy-fiber synapses. J Neurophysiol 71:2552-2556.

Yamamoto C, Sawada S, Kamiya H (1992) Enhancement of postsynaptic responsiveness during long-term potentiation of mossy fiber synapses in guinea pig hippocampus. Neurosci Lett 138:111-114.

Yamamoto C, Sawada S, Ohno-Shosaku T (1993) Quantal analysis of modulating action of adenosine on the mossy fiber synapse in hippocampal slices. Hippocampus 3:87-92.

Yamamoto N, Kurotani T, Toyama K (1989) Neural connections between the lateral geniculate nucleus and visual cortex in vitro. Science 245:192-194.

Yeckel MF, Kapur A, Johnston D (1999) Multiple forms of LTP in hippocampal CA3 neurons use a common postsynaptic mechanism. Nat Neurosci 2:625-633.

Yuste R, Majewska A, Cash SS (1999) Mechanisms of calcium influx into hippocampal spines: heterogeneity among spines, coincidence detection by NMDA receptors, and optical quantal analysis. J Neurosci 19:1976-1987.

Zalutsky RA, Nicoll RA (1990) Comparison of two forms of long-term potentiation in single hippocampal neurons. Science 248:1619-1624.

Zhai RG, Vardinon-Friedman H, Cases-Langhoff C, Becker B, Gundelfinger ED, Ziv NE, Garner CC (2001) Assembling the presynaptic active zone: a characterization of an active zone precursor vesicle. Neuron 29:131-143.

Zucker RS (1999) Calcium- and activity-dependent synaptic plasticity. Curr Opin Neurobiol 9:305-313. 